\title{
ON THE INFIMUM OF THE HAUSDORFF AND VIETORIS TOPOLOGIES
}

\author{
S. LEVI, R. LUCCHETTI, AND J. PELANT
}

(Communicated by Franklin D. Tall)

Dedicated to Professor L. Vietoris on the occasion of his 100th birthday

\begin{abstract}
We study the infimum of the Hausdorff and Vietoris topologies on the hyperspace of a metric space. We show that this topology coincides with the supremum of the upper Hausdorff and lower Vietoris topologies if and only if the underlying metric space is either totally bounded or is a UC space.
\end{abstract}

\section{INTRODUCTION}

The two best known topologies on the hyperspace of a metric space $(X, d)$ are the Hausdorff and Vietoris topologies, denoted by $H$ and $V$ respectively. They were introduced in the twenties and since have been extensively studied (see, e.g., [Mi, Ku, KT]). 3.2]:

Let us recall the result that directly compares the two topologies [Mi, Lemma

$H$ is weaker than $V$ if and only if $(X, d)$ is totally bounded, and $V$ is weaker than $H$ if and only if $(X, d)$ has the UC property; i.e., disjoint closed sets are a positive distance apart.

In this article we wish to study the infimum topology $H \wedge V$, that is, the collection of all subsets of the hyperspace $c(X)$ which are open for both topologies $H$ and $V$.

It is well known that $H$ and $V$ can be split into a lower part, denoted by $H^{-}$and $V^{-}$, respectively, and an upper part, $H^{+}$and $V^{+}$(see the definitions below). Thus a formal application of the distributive laws to $H \wedge V$, written as $\left(H^{+} \vee H^{-}\right) \wedge\left(V^{+} \vee V^{-}\right)$, yields the topology $H^{+} \vee V^{-}$. The latter is the proximal topology that was introduced and studied in [BLLN]; it is easy to show that $H \wedge V \geq H^{+} \vee V^{-}$and that equality holds if $(X, d)$ is either totally bounded or a UC space, that is, if $H$ and $V$ are comparable.

We note that some results on suprema of hyperspace topologies are available in the literature; for example, the Vietoris topology is the supremum over all

Received by the editors October 30, 1991.

1991 Mathematics Subject Classification. Primary 54B20, 54E35. 
equivalent metrics of the corresponding Wijsman, as well as proximal, topologies [BLLN, Theorem 3.1, Corollary 3.5]; also, the supremum of all Hausdorff metric topologies coincides with the so-called locally finite topology [BHPV].

In contrast, there are, to our knowledge, no results concerning the infimum operator which is not as flexible as the supremum, the main reason being that a net converges for the supremum of two topologies if and only if it converges for both, while the dual characterization does not hold for infima; this is an intrinsic difficulty in the lattice of topologies considered as a sublattice of the lattice of convergences.

We will begin our study with an exploration of some particular sets in $H \wedge V$ in relation to $H^{+} \vee V^{-}$. In particular, we show that if a finite union of $V$-basic open sets is $H$-open, then this union belongs to $H^{+} \vee V^{-}$. More interestingly we also prove that all elements of $H \wedge V$ of the form $\bigcup_{\alpha}\left(G_{\alpha}^{+} \cap V_{\alpha}^{-}\right)$, where the $G_{\alpha}$ 's and $V_{\alpha}$ 's are open subsets of $X$, actually belong to $H^{+} \vee V^{-}$.

Our main result, however, states that these two topologies coincide if and only if $(X, d)$ is either totally bounded or UC. This shows how stringent the restriction to a subbase for $V^{-}$is; it also shows that the lattice generated by $\mathrm{H}^{+}, \mathrm{V}^{+}, \mathrm{H}^{-}, \mathrm{V}^{-}$is not distributive in general.

This result provides a good example of the interplay between $X$ and the lattice of topologies on $c(X)$.

Let us recall some definitions. The hyperspace of $X$, denoted by $c(X)$, is the collection of all closed nonempty subsets of $X$. The upper Vietoris topology $V^{+}$on $c(X)$ has for a base the family of all sets of the form $G^{+}=\{F \in c(X)$ : $F \subset G\}$, where $G$ is open in $X$, while the lower Vietoris topology $V^{-}$has for a subbase all sets of the form $G^{-}=\{F \in c(X): F \cap G \neq \varnothing\}$, where $G$ is open in $X$. The Vietoris topology $V$ is the supremum of $V^{+}$and $V^{-}$and a basic open set for $V$ has the form $G^{+} \cap W_{1}^{-} \cap \cdots \cap W_{k}^{-}$, where $G$ and the $W_{k}$ 's are open subsets of $X$.

We similarly define the upper Hausdorff topology $H^{+}$(we should strictly write $H_{d}^{+}$since the equivalent metrics on $X$ can generate different Hausdorff topologies on the hyperspace, but we will drop the subscript if the metric is fixed) by means of a local base: if $A \in c(X)$, consider the family-ranging over all $\varepsilon>0$ - of the

$$
S_{H^{+}}(A, \varepsilon)=\left\{B \in c(X): B \subseteq A^{\varepsilon}\right\},
$$

where $A^{\varepsilon}$ is the $\varepsilon$-expansion of $A$; in a dual manner we obtain the lower Hausdorff topology $H^{-}$by considering as a local base at $A$ the family of all

$$
S_{H^{-}}(A, \varepsilon)=\left\{B \in c(X): A \subseteq B^{\varepsilon}\right\}, \quad \varepsilon>0 .
$$

The set

$$
S(A, \varepsilon)=S_{H^{+}}(A, \varepsilon) \cap S_{H^{-}}(A, \varepsilon)
$$

is the Hausdorff sphere of radius $\varepsilon$ centered at $A$.

The Hausdorff topology is the supremum of $\mathrm{H}^{+}$and $\mathrm{H}^{-}$, and it is well known that $V^{+} \geq H^{+}$and $H^{-} \geq V^{-}$. For general background on hyperspace topologies, the reader is referred to [FLL].

A metric space $(X, d)$ has the UC property if, for any two disjoint closed subsets $A$ and $B$,

$$
D_{d}(A, B)=D(A, B)=\inf \{d(a, b): a \in A, b \in B\}>0 .
$$


There are several known characterizations of UC spaces (see, e.g., [At, Be, Ra]). We mention here that $(X, d)$ is $\mathrm{UC}$ if and only if every continuous real function on $X$ is uniformly continuous and that every UC space is complete.

\section{THE TOPOLOGY $H \wedge V$}

We will say that the open subset $G$ of $X$ has property $(*)$ if $D\left(A, G^{\mathrm{c}}\right)>0$ for every closed subset $A$ of $G$, where $G^{\mathrm{c}}$ is the complement of $G$.

Theorem 1. The following conditions are equivalent.

(i) $V \leq H$.

(ii) $V^{+}=H^{+}$.

(iii) Every open subset of $X$ has property $(*)$.

(iv) $(X, d)$ is a UC space.

Proof. (i) $\Rightarrow$ (ii) Let $G$ be open in $X$; we will show that the basic $V^{+}$-open set $G^{+}$is open in $H^{+}$. We know that $G^{+} \in H$ by (i). Pick $A \in G^{+}$and $\varepsilon>0$ such that $S(A, \varepsilon) \subseteq G^{+}$; then $S_{H^{+}}(A, \varepsilon) \subseteq G^{+}$for if $B \subseteq A^{\varepsilon}, A \cup B$ is in $S(A, \varepsilon)$ and thus in $G^{+}$. Notice that each $G^{+}$has the property that if $C \in G^{+}$ and $D \subseteq C$, then $D \in G^{+}$. Therefore, $B \in G^{+}$and $S_{H^{+}}(A, \varepsilon) \subseteq G^{+}$.

(ii) $\Rightarrow$ (i) follows from the fact that $V^{-} \leq H^{-}$.

(ii) $\Rightarrow$ (iii) Suppose there is $G$ open in $X$ which does not have property (*); then there is $A$ closed and contained in $G$ with $D\left(A, G^{\mathrm{c}}\right)=0$. Thus, for every $\varepsilon>0, A^{\varepsilon}$ is not contained in $G$ and $G^{+}$is not in $H^{+}$.

(iii) $\Rightarrow$ (iv) If $A$ and $B$ are disjoint closed sets, $A \subseteq B^{\mathrm{c}}$ and $D(A, B)>0$.

(iv) $\Rightarrow$ (ii) If $G$ is open and $A \in G^{+}, A$ and $G^{\mathrm{c}}$ are disjoint closed sets. Thus, $D\left(A, G^{\mathrm{c}}\right)=\sigma>0$ and $S_{H^{+}}(A, \varepsilon) \subseteq G^{+}$for $\varepsilon<\sigma$.

As was pointed out previously, the equivalence of (i) and (iv) can be found, with a different proof, in [Mi]. Note that Theorem 3.3 of [BHPV] gives a condition equivalent to (iv) in terms of the locally finite topology.

Corollary 2. A metric space $(X, d)$ has an equivalent $U C$ metric if and only if the set $\left\{H_{\rho}^{+}: \rho\right.$ is a metric equivalent to $\left.d\right\}$ has a maximum in the lattice of topologies on $c(X)$. This maximum is then necessarily equal to $V^{+}$.

Proof. From Corollary 3.5 of [BLLN] we obtain

$$
V^{+}=\sup \left\{H_{\rho}^{+}: \rho \text { is equivalent to } d\right\} .
$$

The corollary is then a consequence of Theorem 1.

The preceding condition extends the list of topological characterizations of the UC property (see, in particular, [Ra]).

Corollary 2 should also be compared to Corollary 5.6 of [BLLN] which states that compactness of $X$ is equivalent to $V^{+}$being equal to the maximum of the set $\left\{W_{\rho}^{+}: \rho\right.$ is equivalent to $\left.d\right\}$, where $W_{\rho}^{+}$is the upper part of the Wijsman topology corresponding to $\rho$; i.e., the net $\left(A_{i}\right) W_{\rho}^{+}$-converges to $A$ if $\rho(x, A) \leq \liminf \rho\left(x, A_{i}\right)$ for every $x$ in $X$.

Remark 3. It can be checked easily that a necessary condition for an open set with nonempty boundary to have property $(*)$ is that its boundary be compact.

The following is an example of an open set $G$ with compact boundary which does not have property $(*)$ or, equivalently, such that $G^{+}$is not in $H^{+}$. Let 
$X$ be the subset of $R^{3}$ given by

$$
X=\{(x, y, z):|y| \leq 1, z=0\} \cup\{(x, y, z): y=0, x \cdot z=1\} .
$$

Put $G=\{(x, y, z):|y| \leq 1, x>0, z=0\}$, and let $A=\{(x, y, z): y=z=$ $0, x \geq 1\}$. Then $A$ is closed in $G$ and its distance to $G^{\mathrm{c}}$ is zero. Notice that the boundary of $G$ is compact nonempty and that its distance to $A$ is one.

Simple examples show that if the boundary of $G$ is empty, $G$ may or may not have property $(*)$.

Looking at lower parts we have

Lemma 4. Let $A \in c(X)$; then $S_{H^{-}}(A, \varepsilon)$ belongs to $V^{-}$if and only if $\alpha(A)<$ $\varepsilon$, where $\alpha(A)$ is the ball-measure of noncompactness of $A$.

Proof. Recall that the measure of noncompactness of $A$ is given by

$$
\alpha(A)=\inf \left\{\varepsilon>0: \exists x_{1}, \ldots, x_{n} \in A \text { and } A \subseteq \bigcup B\left(x_{i}, \varepsilon\right)\right\},
$$

where $B(x, \varepsilon)$ is the open sphere of radius $\varepsilon$ around $x$. Suppose now that $S=S_{H^{-}}(A, \varepsilon)$ belongs to $V^{-}$; then there exist $W_{1}, \ldots, W_{k}$ open in $X$ such that $A \in \cap W_{i}^{-} \subseteq S$. Pick $b_{i} \in A \cap W_{i}$ and put $B=\left\{b_{1}, \ldots, b_{k}\right\} ;$ then $B \subseteq A$ and $B \in \bigcap W_{i}^{-}$. Thus, $B \in S, A \subseteq B^{\varepsilon}$, and $\alpha(A)<\varepsilon$.

If conversely $\alpha(A)<\varepsilon$, there are $0<\sigma<\varepsilon$ and $a_{1}, \ldots, a_{n}$ in $A$ such that $A \subseteq \bigcup B\left(a_{i}, \sigma\right)$. Put $W_{i}=B\left(a_{i}, \varepsilon-\sigma\right)$; then $A \in \bigcap W_{i}^{-}$and if $B \in \bigcap W_{i}^{-}$, $A \subseteq B^{\varepsilon}$.

Theorem 5. The following conditions are equivalent.

(i) $H \leq V$.

(ii) $H^{-}=V^{-}$.

(iii) $(X, d)$ is totally bounded.

Proof. (i) $\Rightarrow$ (ii) Take $U \in H^{-}$and $A \in U . U$ has the property that if $A \in U$ and $B \supseteq A$, then $B \in U$. Since $A \in U \in V$ by (i), there are $G, W_{1}, \ldots, W_{k}$ open in $X$ such that $A \in G^{+} \cap W_{1}^{-} \cap \cdots \cap W_{k}^{-} \subseteq U$. We will show that the $V^{-}$-neighborhood $\left(W_{1} \cap G\right)^{-} \cap \cdots \cap\left(W_{k} \cap G\right)^{-}$of $A$ is contained in $U$. Pick $B \in \bigcap\left(W_{i} \cap G\right)^{-}$and $b_{i} \in W_{i} \cap G$; the closed set $\left\{b_{1}, \ldots, b_{k}\right\} \in G^{+} \cap W_{1}^{-} \cap \cdots \cap W_{k}^{-} \subseteq U$. Thus, its superset $B$ also belongs to $U$.

(ii) $\Rightarrow$ (i) follows from the fact that $H^{+} \leq V^{+}$.

(ii) $\Rightarrow$ (iii) Apply Lemma 4 to $X$.

(iii) $\Rightarrow$ (ii) If $(X, d)$ is totally bounded, each subset has measure of noncompactness equal to zero and, by Lemma 4 , each set $S_{H^{-}}(A, \varepsilon)$ belongs to $V^{-}$.

Corollary 6. A metrizable space $X$ is separable if and only if

$$
V^{-}=\min \left\{H_{\rho}^{-}: \rho \text { is an equivalent metric on } X\right\} .
$$

This follows from Theorem 5 , the fact that $V^{-} \leq H_{\rho}^{-}$for every equivalent $\rho$, and the well-known result that $X$ is separable if and only if it admits a totally bounded metric [En, Theorem 4.3.5].

We now prove a finite absorption property for the topologies $V^{+}$and $\mathrm{H}^{+}$ which states that if a finite union of $V^{+}$-basic open sets belongs to $H^{+}$, it then reduces to the union of the elements already in $\mathrm{H}^{+}$. 
Proposition 7. Suppose $G_{1}, \ldots, G_{n}$ are open subsets of $X$ and that $\cup G_{i}^{+} \in$ $H^{+}$. If, for some $i, G_{i}$ does not have property $(*)$, there exists $j \neq i$ such that $G_{j} \supset G_{i}$ and $G_{j}$ has property $(*)$.

Proof. There exists $A \subset G_{i}$ with $D\left(A, G_{i}^{\mathrm{c}}\right)=0$. If, for every $j \neq i, G_{j}$ does not contain $G_{i}$, pick a point $x_{j}$ in $G_{i}-G_{j}$ and consider the set $B=$ $A \cup\left\{x_{1}, \ldots, x_{n}\right\}$. Then $B \in G_{i}^{+}$, but for no $\varepsilon>0$ does $B^{\varepsilon}$ belong to $\bigcup G_{i}^{+}$, which is a contradiction. Thus there is $j$ with $G_{j} \supset G_{i}$. Suppose further that, for every $k \neq j, G_{j}$ is not contained in $G_{k}$ (this is no restriction since the cardinality of the $i$ 's is finite). Then $G_{j}$ has property $(*)$-if not we can repeat the same argument as above and arrive at a contradiction.

We note that the absorption property is not valid for infinite families. In the plane consider $G_{n}=\{(x, y): y<n\}$. Then no $G_{n}$ has property $(*)$ since its boundary is not compact, but $\bigcup G_{n}^{+}$belongs to $H^{+}$.

The next lemma, valid for any cardinality, has a straightforward proof.

Lemma 8. Suppose $\bigcup_{i}\left(G_{i}^{+} \cap W_{i, 1}^{-} \cap \cdots \cap W_{i, k(i)}^{-}\right)$belongs to $H$. Then $\bigcup_{i} G_{i}^{+}$ belongs to $\mathrm{H}^{+}$.

Corollary 9. If a finite union of $V$-basic elements belongs to $H$, at least one of them belongs to $H$. $H^{-}$.

For the proof first apply Lemma 8, then Proposition 7, and recall that $V^{-} \leq$

Simple examples show that no absorption property holds for finite unions of $V$-basic elements.

Lemma 10. The inequality $H \wedge V \geq H^{+} \vee V^{-}$holds in a general metric space. If $(X, d)$ is either totally bounded or UC, equality holds.

Proof. Since $H^{+} \vee V^{-}$is weaker than both $H$ and $V$, the inequality follows. If $(X, d)$ is UC, then, by Theorem $1, H \wedge V=V=H^{+} \vee V^{-}$. If $(X, d)$ is totally bounded, then, by Theorem $5, H \wedge V=H=H^{+} \vee V^{-}$.

Theorem 11. Let $O_{1}, \ldots, O_{n}$ be basic open sets for the Vietoris topology and suppose $\bigcup_{i} O_{i}$ is $H$-open. Then $\bigcup_{i} O_{i}$ belongs to $\mathrm{H}^{+} \vee V^{-}$.

Proof. Let $A \in \bigcup_{i} O_{i}$. We must show that there is a neighborhood of $A$ for $H^{+} \vee V^{-}$which is contained in $\bigcup_{i} O_{i}$. Since this union is $H$-open, there is $\varepsilon>0$ such that $S(A, \varepsilon) \subset \bigcup_{i} O_{i}$. Put

$$
N=\left(A^{\varepsilon}\right)^{+} \cap W_{1}^{-} \cap \cdots \cap W_{j}^{-},
$$

where $\left\{W_{1}, \ldots, W_{j}\right\}$ is the family of all open subsets of $X$ that are part of the definition of the lower parts of the $O_{i}$ 's and that intersect $A$. Then $N$ is an $\left(H^{+} \vee V^{-}\right)$-neighborhood of $A$. If $B \in N$ we notice that $A \cup B \in S(A, \varepsilon)$; therefore, $A \cup B \in O_{k}$ for some $k$ and $A \cup B$ intersects the $W_{i}$ 's that define $O_{k}$. $B$ intersects all of them since if $A$ intersects one of them then so does $B$ because $B \in N$ and if $A$ does not then $B$ will because $A \cup B$ meets it. Hence $B \in O_{k}$ and the result is proved.

As we will see, this result does not extend to infinite families, but, surprisingly enough, it does if we restrict ourselves to subbasic open sets for the lower parts. 
Theorem 12. Suppose that, for each $i, O_{i}=G_{i}^{+} \cap W_{i}^{-}$, where $G_{i}$ and $W_{i}$ are open subsets of $X$ and that $\bigcup_{i} O_{i}$ is $H$-open. Then $\bigcup_{i} O_{i}$ belongs to $H^{+} \vee V^{-}$.

Proof. Let $A \in \bigcup_{i} O_{i}$ and consider the following partition of the indices:

$i$ is an $\alpha$-index if $A \in O_{i}$.

$i$ is a $\beta$-index if $A \subset G_{i}$ and $A$ does not meet $W_{i}$.

$i$ is a $\gamma$-index if $A$ is not contained in $G_{i}$.

Since $\bigcup_{i} O_{i}$ is $H$-open, there is $\varepsilon>0$ such that this union contains $S(A, \varepsilon)$. Pick $0<\sigma<\varepsilon$; then $\operatorname{cl}\left(A^{\sigma}\right)$ (the closure of $A^{\sigma}$ ) belongs to $O_{i_{0}}$ for some $i_{0}$. If $A$ meets $W_{i_{0}}$, that is, $i_{0}$ is an $\alpha$-index, say $\alpha_{0}$, we consider the $\left(H^{+} \vee V^{-}\right)$neighborhood of $A$ given by $N=\left(A^{\sigma}\right)^{+} \cap W_{\alpha_{0}}^{-}$; then every $B$ in $N$ belongs to $O_{\alpha_{0}}$. If $A$ does not meet $W_{i_{0}}$, that is, $i_{0}$ is a $\beta$-index since $A \subset G_{i_{0}}$, put $i_{0}=\beta_{1}$ and consider $H_{1}=\operatorname{cl}\left(A^{\sigma}\right)-W_{\beta_{1}} ; H_{1}$ is a closed set such that $A \subseteq H_{1} \subset A^{\varepsilon}$; therefore, $H_{1} \in S(A, \varepsilon)$ and $H_{1} \in O_{i_{1}}$ for some $i_{1}$. If $i_{1}$ is an $\alpha$-index, say $\alpha_{1}$, we consider the $\left(H^{+} \vee V^{-}\right)$-neighborhood of $A$ given by $N=\left(A^{\sigma}\right)^{+} \cap W_{\alpha_{1}}^{-}$and show that $N \subseteq \bigcup_{i} O_{i}$. Indeed if $B \in N$ and $B$ meets $W_{\beta_{1}}$, then $B \in O_{\beta_{1}}$ since $A^{\sigma} \subseteq G_{\beta_{1}}$; if $B$ does not meet $W_{\beta_{1}}$, then $B \subseteq H_{1} \subseteq G_{\alpha_{1}}$ and $B \in O_{\alpha_{1}}$; if $i_{1}$ is a $\beta$-index (it cannot be a $\gamma$-index), call it $\beta_{2}$ and notice that $\beta_{2} \neq \beta_{1}$ since $H_{1} \cap W_{\beta_{2}}$ is not empty while $H_{1} \cap W_{\beta_{1}}$ is. Consider now $H_{2}=\operatorname{cl}\left(A^{\sigma}\right)-\left(W_{\beta_{1}} \cup W_{\beta_{2}}\right)$; this again is a closed set in $S(A, \varepsilon)$ and, therefore, belongs to some $O_{i_{2}}$. If $i_{2}$ is an $\alpha$-index, we conclude as above and if $i_{2}$ is a $\beta$-index, say $\beta_{3}$, we notice that $\beta_{3} \neq \beta_{2}$ and $\beta_{3} \neq \beta_{1}$.

We proceed by transfinite induction, supposing that at no step of our construction do we meet an $\alpha$-index. The above procedure holds for every successor ordinal; suppose now $\xi$ is a limit ordinal and consider the closed set $H_{\xi}=\operatorname{cl}\left(A^{\sigma}\right)-\bigcup_{\eta<\xi} W_{\eta}$, knowing that $A$ does not meet any of the $W_{\eta}$ 's. Then $H_{\xi}$ belongs to some $O_{i}$ and we can put $i=\beta_{\xi}$. The mapping constructed in this way is an injective map from an initial segment of the ordinals, of cardinality equal to the cardinality of the set of $\beta$-indices, into the set of $\beta$-indices. In this way we exhaust the $\beta$-indices and, therefore, must obtain an $\alpha$-index at some step and the theorem is proved.

Remark 13. Suppose the union $\bigcup_{i} O_{i}$ of general $V$-basic open sets is $H$-open and that $A \in \bigcup_{i} O_{i}$. It can be shown that $A$ is an interior point of $\bigcup_{i} O_{i}$ for the topology of $\mathrm{H}^{+} \vee V^{-}$in the following two cases:

(i) $A$ meets a finite number of elements defining the lower parts of the $O_{i}$ 's.

(ii) The cardinality of the set of $\beta$-indices is finite.

The proof in case (ii) follows the scheme of Theorem 12.

We now state our main result.

Theorem 14. Let $(X, d)$ be a metric space. Then

$$
H \wedge V=H^{+} \vee V^{-}
$$

if and only if $(X, d)$ is either totally bounded or a UC space.

Proof. Lemma 10 takes care of the if part. We divide the proof of the only if part into two main steps. The first step is aimed at showing that if $X$ is neither totally bounded nor UC, there are three countable closed subsets $A=\left\{a_{n}\right\}$, 
$B=\left\{b_{n}\right\}, C=\left\{c_{n}\right\}$, and $\sigma>0$ with the following properties:

(1) $A \cap B=\varnothing$ and $d\left(a_{n}, b_{n}\right) \rightarrow 0$.

(2) $d\left(c_{i}, c_{j}\right) \geq 2 \sigma$ for $i \neq j$.

(3) $D(A, C) \geq \sigma, D(B, C) \geq \sigma$.

If $X$ is not UC, we can find sequences $A$ and $B$ with distinct points and with property (1). If $X$ is not totally bounded, we can find a sequence $C$ with (2). Now observe that conditions (1) and (2) still hold if we pass to subsequences of $A, B$, and $C$. Thus if $D(A, C)>0$, we are done; then suppose $D(A, C)=$ 0 . Without loss of generality we can suppose that $A \cap C=\varnothing$ (if $A \cap C$ is countable, assign to $A$ the even subsequence and to $C$ the odd one). Then there are two subsequences, labeled by $n,\left\{a_{n}\right\}$ of $A$ and $\left\{c_{n}\right\}$ of $C$ such that $d\left(a_{n}, c_{n}\right) \rightarrow 0$; then $d\left(b_{n}, c_{n}\right) \rightarrow 0$ because of $(1)$. Thus there is $k$ such that, for all $i, j>k, d\left(c_{2 i+1}, a_{2 j}\right) \geq \sigma$ and $d\left(c_{2 i+1}, b_{2 j}\right) \geq \sigma$.

Put $A^{\prime}=\left\{a_{2 n}: n>k\right\}, B^{\prime}=\left\{b_{2 n}: n>k\right\}$, and $C^{\prime}=\left\{c_{2 n+1}: n>k\right\}$. Then $A^{\prime}, B^{\prime}$, and $C^{\prime}$ verify (1), (2), and (3). Call them $A, B$, and $C$.

The second step in the proof consists in showing that the existence of the sets $A, B$, and $C$ implies that $H \wedge V$ is strictly stronger than $H^{+} \vee V^{-}$.

To do this we produce a set $O$ which is $(H \wedge V)$-open but not $\left(H^{+} \vee V^{-}\right)$open. Let us put

$$
A_{n}=\left\{a_{i}: i \leq n\right\}, \quad C_{n}=\left\{c_{i}: i \leq n\right\}, \quad B_{n}=\left\{b_{i}: i>n\right\}
$$

and define, for every $n, F_{n}=A_{n} \cup C_{n} \cup B_{n}$. It is easy to check that the sequence $\left\{F_{n}\right\}$ is $\left(H^{+} \vee V^{-}\right)$-convergent to $A \cup C$; thus the set $O=c(X)-\left\{F_{n}: n \in N\right\}$ is not $\left(H^{+} \vee V^{-}\right)$-open. The proof will be complete if we show that $O$ is $(H \wedge V)$-open. We will prove that every $K$ in $O$ has $V$-and $H$-neighborhoods which do not contain the $F_{n}$ 's for all large $n$ 's (this is sufficient since $H$ and $V$ are Hausdorff topologies). We will consider the following three exhaustive cases:

(a) There is $x \in K-(A \cup C)$.

(b) There is $x \in(A \cup C)-K$.

(c) $K=A \cup C$.

In case (a) let $0<\varepsilon$ be such that $B(x, 2 \varepsilon) \cap(A \cup C)=\varnothing$ and put $S=$ $B(x, \varepsilon)$. Then $K \in S^{-}$and since, for large $n, d\left(b_{n}, A\right)<\varepsilon$, the set $B_{n}$ must be disjoint from $S$. Thus, for large $n, F_{n}$ does not meet $S$ and $S^{-}$acts as both $H$ - and $V$-neighborhood of $K$.

In case (b) let $0<\varepsilon$ be such that $B(x, 2 \varepsilon) \cap K=\varnothing$; since $x \in F_{n}$ for all large $n, S_{H^{+}}(K, \varepsilon)$ does not contain the corresponding $F_{n}$ 's and $S_{H^{+}}(K, \varepsilon)$ acts as both $H$ - and $V$-neighborhood of $K$.

In case (c) notice first that, for $0<\varepsilon<\sigma, S_{H^{-}}(A \cup C, \varepsilon)$ does not contain any $F_{n}$. For each $n$, pick $c_{k}$ in $C$ with $k>n$. Then $d\left(c_{k}, C_{n}\right) \geq 2 \sigma$ by (2), $d\left(c_{k}, A_{n}\right) \geq d\left(c_{k}, A\right) \geq D(A, C) \geq \sigma>\varepsilon$, and $d\left(c_{k}, B_{n}\right) \geq D(B, C) \geq \sigma>\varepsilon ;$ thus, $c_{k}$ does not belong to $\left(F_{n}\right)^{\varepsilon}$ and $S_{H^{-}}(A \cup C, \varepsilon)$ is the required $H$ neighborhood of $A \cup C$. As for the $V$-neighborhood, notice that for each $n$ there is $\varepsilon_{n}>0$ such that $(A \cup C \cup B) \cap B\left(a_{n}, \varepsilon_{n}\right)=\left\{a_{n}\right\}$; thus the open set $G=\bigcup_{n} B\left(a_{n}, \varepsilon_{n}\right) \cup \bigcup_{n} B\left(c_{n}, \varepsilon\right)$, with $\varepsilon<\sigma$, contains $A \cup C$ but no $F_{n}$. Therefore, $G^{+}$is a $V$-neighborhood of $A \cup C$ contained in $O$ and the proof of the theorem is complete. 
We close the paper with some remarks on subspaces. Let $F$ be a closed subspace of $X$. It can be checked that the topologies $H^{+}, H^{-}, V^{+}, V^{-}$on $c(F)$, the hyperspace of $F$, agree with the restriction to $c(F)$ of the corresponding ones defined on $X$. The symbols $H_{F}^{+}, V_{F}^{-}$, and similar ones are therefore unambiguously defined, as well as $H_{F}$ and $V_{F}$ in virtue of

Lemma 15. The supremum and restriction operators commute.

We note that we also have

Lemma 16. $(H \wedge V)_{F}=H_{F} \wedge V_{F}$ for every closed subspace $F$ of $X$.

Proof. It is clear that $(H \wedge V)_{F} \leq H_{F} \wedge V_{F}$, and to show the reverse inequality let $G \in H_{F} \wedge V_{F}$; define

$$
G^{*}=G \cup\{P \in c(X): P-F \neq \varnothing\} .
$$

Then $G^{*} \in H \wedge V$ and $c(F) \cap G^{*}=G$.

Recently the bounded Hausdorff and bounded Vietoris hyperspace topologies were introduced and investigated (see, e.g., [BL] and the references therein). It can be shown, with techniques similar to those developed in this paper, that the lattice generated by the bounded Hausdorff and Vietoris (or bounded Vietoris) topologies is distributive if and only if the two topologies are comparable.

\section{REFERENCES}

[At] M. Atsuji, Uniform continuity of continuous functions of metric spaces, Pacific J. Math. 8 (1958), 11-16.

[Be] G. Beer, Metric spaces on which continuous functions are uniformly continuous and Hausdorff distance, Proc. Amer. Math. Soc. 95 (1985), 653-658.

[BHPV] G. Beer, C. J. Himmelberg, K. Prikry, and F. S. Van Vleck, The locally finite topology on $2^{X}$, Proc. Amer. Math. Soc. 101 (1987), 168-172.

[BL] G. Beer and R. Lucchetti, Weak topologies for the closed subsets of a metrizable space, Trans. Amer. Math. Soc. (to appear).

[BLLN] G. Beer, A. Lechicki, S. Levi, and S. Naimpally, Distance functionals and suprema of hyperspace topologies, Ann. Mat. Pura Appl. (4) (to appear).

[En] R. Engelking, General topology, vol. 60, PWN, Warsaw, 1977.

[FLL] S. Francaviglia, A. Lechicki, and S. Levi, Quasi-uniformization of hyperspaces and convergence of nets of semicontinuous multifunctions, J. Math. Anal. Appl. 112 (1985), 347-370.

[Mi] E. Michael, Topologies on spaces of subsets, Trans. Amer. Math. Soc. 71 (1951), 152-182.

[KT] E. Klein and A. Thompson, Theory of correspondences, Wiley, New York, 1984.

[Ku] K. Kuratowski, Topology, vol. 1, Academic Press, New York, 1966.

[Ra] J. Rainwater, Spaces whose finest uniformity is metric, Pacific J. Math. 9 (1959), 567-570.

(S. Levi and R. Lucchetti) Dipartimento di Matematica, Università di Milano, Via Saldini 50, 20133 Milano, Italy

e-mail,S. Levi: slevi@vmimat.mat.unimi.it

e-mail, R. Lucchetti: rel@vmimat.mat.unimi.it

(J. Pelant) Mathematicky Ustav, Czechoslovak Academy of Sciences, Zitna 25, 11567 Praha, Czechoslovakia

E-mail address: pelant@csearn.bitnet 